\title{
On the Corona of Two Graphs ${ }^{1}$ )
}

Roberto Frucht and Frank Harary (Valparaiso, Chile; Ann Arbor, Mich., U.S.A.)

Our object in this note is to construct a new and simple operation on two graphs $G_{1}$ and $G_{2}$, called their corona, with the property that the group of the new graph is in general isomorphic with the wreath product of the groups of $G_{1}$ and of $G_{2}$.

Consider two permutation groups $A$ and $B$ of order $m$ and $n$ respectively acting on objects sets $X=\left\{x_{1}, x_{2}, \ldots, x_{d}\right\}$ and $Y=\left\{y_{1}, y_{2}, \ldots, y_{e}\right\}$. By their composition or wreath product $A[B]$ we will mean that permutation group of order $m n^{d}$ acting on $X \times Y$ in which each permutation $\alpha \in A$ and each sequence $\left(\beta_{1}, \beta_{2}, \ldots, \beta_{d}\right)$ of permutations in $B$ induce the permutation $\gamma=\left(\alpha ; \beta_{1}, \beta_{2}, \ldots, \beta_{d}\right)$ such that $\gamma\left(x_{i}, y_{j}\right)=\left(\alpha x_{i}, \beta_{i} y_{j}\right)$. We write $A \equiv B$ to mean that two permutation groups $A$ and $B$ are not only isomorphic but also permutationally equivalent. More specifically let $h: A \leftrightarrow B$ be an isomorphism. To define $A \equiv B$, we also require another $1-1$ map $f: X \leftrightarrow Y$ between the objects such that for all $x$ in $X$ and $\alpha$ in $A, f(\alpha x)=h(\alpha) f(x)$.

Let graphs') $G_{1}$ and $G_{2}$ have point sets $V_{1}$ and $V_{2}$. Then $G_{1}\left[G_{2}\right]$, their lexicographic product, has $V_{1} \times V_{2}$ as its set of points, with two of its points $u=\left(u_{1}, u_{2}\right)$ and $v=\left(v_{1}, v_{2}\right)$ adjacent whenever $u_{1}$ is adjacent to $v_{1}$ in $G_{1}$, or $u_{1}=v_{1}$ and $u_{2}$ is adjacent to $v_{2}$ in $G_{2}$. Let $A_{1}$ and $A_{2}$ be the groups of graphs $G_{1}$ and $G_{2}$; then necessary and sufficient conditions for $A_{1}\left[A_{2}\right]$ to be permutationally equivalent with the group of $G_{1}\left[G_{2}\right]$ were found by Sabidussi [2]. In order to state this result, we recall from [1] that $\Gamma(G)$ denotes the group of graph $G$ and $\bar{G}$ the complement of $G$, that the neighborhood $N(v)$ of a point $v$ of $G$ is the set of points adjacent with $v$, and the closed neighborhood of $v$ is $N(v) \cup\{v\}$.

THEOREM (Sabidussi). If $G_{1}$ is not totally disconnected, then the group of the lexicographic product of two graphs $G_{1}$ and $G_{2}$ is the wreath product of their groups,

$$
\Gamma\left(G_{1}\left[G_{2}\right]\right) \equiv \Gamma\left(G_{1}\right)\left[\Gamma\left(G_{2}\right)\right]
$$

if and only if the following two conditions hold:

1) If there are two points in $G_{1}$ with the same neighborhood, then $G_{2}$ is connected.

1) Research supported in part by grants from the government of Chile and the U.S. Air Force Office of Scientific Research.

2) The definitions of graphical concepts not included here may be found in the book [1]. In particular, a graph $G$ has a finite nonempty set $V$ of points and a set of lines which is a subset of all unordered pairs of points. 
2) If there are two points in $G_{1}$ with the same closed neighborhood, then $\bar{G}_{2}$ is connected.

These conditions for the group of the lexicographic products of two graphs to be permutationally equivalent to the composition of their groups are rather complex. This suggests that another operation on graphs be constructed for the purpose of realizing the composition of their groups only up to group isomorphism.

The corona $G_{1} \bigcirc G_{2}$ of two graphs $G_{1}$ and $G_{2}$ (where $G_{i}$ has $p_{i}$ points and $q_{i}$ lines) is defined as the graph $G$ obtained by taking one copy of $G_{1}$ and $p_{1}$ copies of $G_{2}$, and then joining by a line the $i$ 'th point of $G_{1}$ to every point in the $i$ 'th copy of $G_{2}$. It follows from the definition of the corona that $G_{1} \bigcirc G_{2}$ has $p_{1}\left(1+p_{2}\right)$ points and $q_{1}+p_{1} q_{2}+p_{1} p_{2}$ lines. This fact allows us to recognize immediately that the associative law never holds: $G_{1} \bigcirc\left(G_{2} \circ G_{3}\right)$ and $\left(G_{1} \circ G_{2}\right) \circ G_{3}$ are always different graphs. Indeed the first one has $p_{1}\left(1+p_{2}+p_{2} p_{3}\right)$ points while the second has $p_{1}\left(1+p_{2}\right)\left(1+p_{3}\right)$ points, and these numbers are never equal.

We denote the identity group of degree 1 (and order 1) by $E_{1}$ and define the sum $A+B$ of two permutation groups $A$ and $B$ as follows. The group $A+B$ acts on the disjoint union $X \cup Y$ and its elements are all the ordered pairs, written $\alpha+\beta$, of permutations $\alpha \in A, \beta \in B$, given by

$$
(\alpha+\beta) z= \begin{cases}\alpha z, & z \in X \\ \beta z, & z \in Y\end{cases}
$$

Note that the trivial graph $K_{1}$ consists of one isolated point (of degree zero).

THEOREM 1. The group of the corona of two graphs $G_{1}$ and $G_{2}$ can be written explicitly in terms of the following wreath product involving their groups,

$$
\Gamma\left(G_{1} \bigcirc G_{2}\right) \equiv \Gamma\left(G_{1}\right)\left[E_{1}+\Gamma\left(G_{2}\right)\right],
$$

if and only if at least one of the two graphs $G_{1}$ and $\vec{G}_{2}$ has no isolated points.

Proof. Automorphisms $\gamma$ of the corona $G_{1} \circ G_{2}$ may be obtained by the action of one automorphism $\alpha_{1}$ of $G_{1}$ combined with $p_{1}$ automorphisms $\beta_{i}$ of the graph $H_{2}$ obtained from $G_{2}$ by adding a new point $w$ to $G_{2}$ and joining $w$ by lines with every point of $G_{2}$, such that every automorphism $\beta_{i}$ leaves $w$ fixed. But we now see that $\gamma$ acts on the set of points of $G_{1} \circ G_{2}$ precisely in accordance with the definition of the wreath product of the two permutation groups $\Gamma\left(G_{1}\right)$ and $E_{1}+\Gamma\left(G_{2}\right)$. For $\alpha_{1}$ permutes the points of $G_{1}$, and hence in the corona graph $G_{1} \odot G_{2}, \alpha_{1}$ permutes the $p_{1}$ copies of $H_{2}$ constructed above. Then each of the $p_{1}$ automorphisms $\beta_{1}, \beta_{2}, \ldots$ permutes one of the copies of $\mathrm{H}_{2}$ in an adjacency preserving manner, and leaves $w$ fixed. Thus the automorphisms considered so far constitute a permutation group on the $p_{1}\left(1+p_{2}\right)$ points of $G_{1} \bigcirc G_{2}$ that is permutationally equivalent to the wreath product $\Gamma\left(G_{1}\right)\left[E_{1}+\Gamma\left(G_{2}\right)\right]$. 
It remains to be shown that if and only if at least one of the graphs $G_{1}$ and $\bar{G}_{2}$ has no isolated points, no further automorphisms of the corona $G_{1} \odot G_{2}$ can exist. Now to prove the necessity of this condition we will show that such an automorphism really does exist if both $G_{1}$ and $\bar{G}_{2}$ have isolated points.

Indeed let $u_{i}$ be an isolated point of $G_{1}$, and $v_{j}$ an isolated point of $\vec{G}_{2}$. Then $v_{j}$ is joined in $G_{2}$ by lines with all the other points of that graph. Now consider in $G_{1} \odot G_{2}$ that copy $G_{2}^{(i)}$ of $G_{2}$ whose points are joined with the point $u_{i}$ of $G_{1}$, and let $v_{j, i}$ be that point of $G_{2}^{(i)}$ that corresponds to the point $v_{j}$ of $G_{2}$. Since then both $u_{i}$ and $v_{j, i}$ are joined by lines with all the other points of $G_{2}^{(i)}$, but with no other points of the corona $G_{1} \bigcirc G_{2}$ (if we except the line joining $u_{i}$ and $v_{j, i}$ ), it is obvious that on interchanging $u_{i}$ and $v_{j, i}$ (but leaving fixed all the other points of $G_{1} \odot G_{2}$ ), one obtains an automorphism of $G_{1} \circ G_{2}$ that does not belong to the wreath product $\Gamma\left(G_{1}\right)\left[E_{1}+\Gamma\left(G_{2}\right)\right]$. Hence in order to exclude the existence of such an automorphism it is necessary that at least one of the graphs $G_{1}$ or $\bar{G}_{2}$ have no isolated points.

It remains to prove the sufficiency of these conditions. In other words we have to show that if

(i) $G_{1}$ has no isolated points, or

(ii) $G_{2}$ has no isolated points, then $G_{1} \bigcirc G_{2}$ does not admit other automorphisms than those of the wreath product $\Gamma\left(G_{1}\right)\left[E_{1}+\Gamma\left(G_{2}\right)\right]$.

Now it is obvious than an automorphism not belonging to the wreath product $\Gamma\left(G_{1}\right)\left[E_{1}+\Gamma\left(G_{2}\right)\right]$ would take at least one point of one of the copies of $G_{2}$ into a point of $G_{1}$. But this is impossible since we will show that if (i) or (ii) holds, in the corona $G_{1} \bigcirc G_{2}$ the degree of any point of $G_{1}$ is always higher than that of any other point.

For this purpose let $r$ be the degree in $G_{1}$ of any point $u_{i}$ of $G_{1}$; its degree in $G_{1} \bigcirc G_{2}$ will then be $r+p_{2}$, as seen immediately from the definition of corona. On the other hand, if a point $v_{j}$ of $G_{2}$ has degree $s$ in $G_{2}$, the degree of the corresponding point $v_{j, k}$ of any copy $G_{2}^{(k)}\left(k=1,2, \ldots, p_{1}\right)$ will evidently be $s+1$. What we have then to show is that the inequality

$$
r+p_{2}>s+1
$$

holds for any pair of points from $G_{1}$ and $G_{2}$ if (i) or (ii) is satisfied.

Proof of (1) for the Case (i). If $G_{1}$ has no isolated points, we have $r>0$ for every point of $G_{1}$. Moreover, since $G_{2}$ is a graph, it follows that

and $r+p_{2}>p_{2} \geqslant s+1$.

$$
s \leqslant p_{2}-1 \text {, }
$$

Proof of (1) for Case (ii). If $\bar{G}_{2}$ has no isolated point, and if

$$
s=p_{2}-1
$$


holds for a point $v$ of $G_{2}$, then $v$ would be joined by lines with all the other points of $G_{2}$, and hence would be an isolated point of $\bar{G}_{2}$. Thus

$$
s<p_{2}-1
$$

for all the points of $G_{2}$. A fortiori, $r+p_{2}>s+1$ (since $r \geqslant 0$ ).

The main result of this note is an immediate consequence of Theorem 1 .

COROLLARY. The group of the corona $G_{1} \circ G_{2}$ of two graphs is isomorphic to the wreath product $\Gamma\left(G_{1}\right)\left[\Gamma\left(G_{2}\right)\right]$ of their groups if and only if at least one of the graphs $G_{1}$ and $\bar{G}_{2}$ has no isolated points.

Finally it might be observed that the corona operation allows us to obtain another graph whose group is in general isomorphic to the wreath product of the groups of the graphs $G_{1}$ and $G_{2}$, a sufficient condition being this time only that $G_{2}$ have no isolated points. For this purpose, consider the graph $H=G_{2} \cup K_{1}$ obtained from $G_{2}$ by adding a new point and no new lines to $G_{2}$. This new graph might be described as the complement of $K_{1} \odot \bar{G}_{2}$. For this graph $H$, the following theorem holds.

THEOREM 2. If $G_{2}$ has no isolated points, then,

$$
\Gamma\left(G_{1} \bigcirc\left(G_{2} \cup K_{1}\right)\right) \equiv \Gamma\left(G_{1}\right)\left[E_{2}+\Gamma\left(G_{2}\right)\right] .
$$

Proof. Let $H=G_{2} \cup K_{1}$; then $\bar{H}=K_{1} \circ \bar{G}_{2}$ is obviously a graph without isolated points, and it follows from Theorem 1 that

$$
\Gamma\left(G_{1} \circ H\right) \equiv \Gamma\left(G_{1}\right)\left[E_{1}+\Gamma(H)\right] .
$$

Now since the group of a graph and that of its complement are permutationally equivalent, we also have

$$
\begin{aligned}
\Gamma\left(G_{1} \bigcirc H\right) & \equiv \Gamma\left(G_{1}\right)\left[E_{1}+\Gamma(\bar{H})\right], \\
\Gamma\left(G_{1} \bigcirc\left(K_{1} \cup G_{2}\right)\right) & \equiv \Gamma\left(G_{1}\right)\left[E_{1}+\Gamma\left(K_{1} \bigcirc G_{2}\right)\right] .
\end{aligned}
$$

Since the complement of $\bar{G}_{2}$, namely $G_{2}$ itself, has no isolated points, it follows from Theorem 1 that

$$
\begin{aligned}
\Gamma\left(K_{1} \odot \overline{\left.G_{2}\right)}\right. & \equiv \Gamma\left(K_{1}\right)\left[E_{1}+\Gamma\left(G_{2}\right)\right] \\
& \equiv E_{1}\left[E_{1}+\Gamma\left(G_{2}\right)\right], \\
& \equiv E_{1}+\Gamma\left(G_{2}\right)
\end{aligned}
$$

by the definition of wreath product.

\section{REFERENCES}

[1] Harary, F., Graph Theory (Addison-Wesley, Reading, Mass. 1969).

[2] Sabidussi, G., The Composition of Graphs, Duke Math. J. 26, 693-696 (1959).

Santa Maria University and University of Michigan 